\title{
Psychische Arbeitsbelastungen in Deutschland: Schwerpunkte - Trends - betriebliche Umgangsweisen
}

\author{
Uwe Lenhardt \\ Michael Ertel \\ Martina Morschhäuser
}

\begin{abstract}
An medialer Aufmerksamkeit für das Thema psychischer Arbeitsbelastungen hat es in der jüngeren Vergangenheit nicht gemangelt. Wenn etwa über die Zunahme psychischer Störungen berichtet wird, kommen vielfach auch Phänomene der modernen Arbeitswelt zur Sprache, seien es wachsender Leistungsdruck, gestiegene Flexibilitätsanforderungen und erhöhte Verantwortungslast, oder sei es die um sich greifende Unsicherheit der beruflichen Stellung und Perspektive. In der Tat existiert inzwischen eine Fülle von wissenschaftlichen Belegen für die - psychischen wie körperlichen - Gesundheitsrisiken, die mit solchen Arbeitsbedingungen verbunden sind. Hinweise auf deren Verbreitung und Entwicklungstendenz liefern großangelegte repräsentative Beschäftigtenbefragungen.
\end{abstract}

\section{1 \\ Einleitung}

Aufgeschreckt durch die Selbstmordserie beim Telefonkonzern France Télécom reagiert die französische Regierung mit einem „Notplan“ gegen Stress am Arbeitsplatz (Süddeutsche Zeitung vom 14.2.2010). In Deutschland sind es vor allem deutlich ansteigende Arbeitsunfähigkeitstage aufgrund psychischer Erkrankungen, die alarmieren und die Frage nach Zusammenhängen zu psychischen Belastungen in der Arbeit aufwerfen (BKK Bundesverband 2008).

Gleichzeitig erlebt der Begriff „Burnout“ hierzulande Hochkonjunktur. „Berufsalltag: Erschöpft und enttäuscht. Ein Drittel der Lehrer leidet unter Burnout", meldet die Süddeutsche Zeitung im April 2009. Nach einer aktuellen Studie des Instituts für sozialwissenschaftliche Forschung (ISF) München fühlt sich jeder zweite Beschäftigte in der IT-Branche überlastet (Boes et al. 2009). Und gemäß einem Befund der Universitätskliniken Hamburg und Düsseldorf klagen $22 \%$ der Chirurgen über negativ erlebten beruflichen Stress, wobei die eigene Überarbeitung nach Meinung von $44 \%$ der Befragten die Qualität der Patientenversorgung gefährde (Knesebeck v.d. et al. 2010). „Die Burn-
out-Gesellschaft", so betitelt der Focus verallgemeinernd sein Schwerpunktthema in der Märzausgabe 2010 und sieht steigende Arbeitsanforderungen und -belastungen als wesentlichen Grund für die „wachsende Zahl der Ausgebrannten“.

Der unmittelbare Rückschluss von psychischen Störungen auf psychische Belastungen am Arbeitsplatz wäre allerdings voreilig. Die registrierte Anzahl psychischer Störungen und deren Entwicklung hängen auch von personenbezogenen und außerberuflichen Faktoren ab sowie von der Art der individuellen und gesellschaftlichen Thematisierung und Behandlung des „Psychischen“. Und umgekehrt gilt: Psychische Belastungen können nicht nur zu psychischen Störungen beitragen, sondern sich sehr unterschiedlich auswirken, etwa in Form von Motivationsverlust und Leistungsabfall oder in Form gesteigerten Medikamenten- und Alkoholkonsums. Außerdem tragen sie zur Entstehung zahlreicher, in der Bevölkerung weit verbreiteter körperlicher Krankheiten wie z.B. Muskel-Skelett- oder Herz-KreislaufErkrankungen bei.

Zusammenhänge zwischen psychischen Belastungen und - psychischen wie nicht psychischen - Gesundheitsproblemen sind empirisch gut belegt (Hasselhorn/Portuné 2010). Zum Nachweis gesundheitlicher Folgen von "Stress“ und zugrunde liegender Wirkungszusam- menhänge haben theoretische Modelle mit langer Forschungstradition, so das Demand-Control-Support-Modell von Karasek/Theorell (1990) oder das Modell beruflicher Gratifikationskrisen von Siegrist (1996), einen wichtigen Beitrag geleistet. Danach hängt das „Risikopotenzial“ hoher Arbeitsanforderungen auch von

\footnotetext{
Uwe Lenhardt, Dr., wissenschaftlicher Mitarbeiter an der Bundesanstalt für Arbeits schutz und Arbeitsmedizin. Arbeitsschwerpunkt: Strategiefragen des Arbeitsschutzes und der Arbeitsschutzforschung. e-mail: lenhardt.uwe@baua.bund.de Michael Ertel, Dipl. Soz., wissenschaftlicher Mitarbeiter an der Bundesanstalt für Arbeitsschutz und Arbeitsmedizin. Arbeitsschwerpunkte: Psychische Belastungen und Stress bei der Arbeit, ganzheitliche Gefährdungsbeurteilung.

e-mail: ertel.michael@baua.bund.de Martina Morschhäuser, Dr., leitet die Gruppe "Psychische Belastungen, Arbeitszeitgestaltung, Betriebliches Gesundheitsmanagement" an der Bundesanstalt für Arbeitsschutz und Arbeitsmedizin. Arbeitsschwerpunkte: Psychische Belastungen, alternsgerechte Arbeits- und Personalpolitik, betriebliches Gesundheitsmanagement. e-mail: morschhaeuser.martina@baua. bund.de
} 
vorhandenen beruflichen wie außerberuflichen Unterstützungsstrukturen und „Ressourcen“ ab, denen also gleichfalls Aufmerksamkeit gebührt.

$\mathrm{Zu}$ berücksichtigen ist ferner, dass psychische Belastungen - zumindest gemäß ihrer „neutralen“ Definition - auch aktivierende, entwicklungsförderliche und damit positive Effekte zeitigen können. Vielfach sind die Wirkungen ambivalent, so z.B., wenn Arbeitsplatzwechsel Lernund Entwicklungsmöglichkeiten bietet, gleichzeitig aber mit einem Verlust von Routinen und sozialer Unterstützung am Arbeitsplatz einhergeht. Somit kommt es entscheidend darauf an, wie sich Arbeitsbelastungen und Ressourcen in ihrer Gesamtheit für den Einzelnen darstellen und wie sie sich über die Zeit hinweg im Erwerbsverlauf gestalten.

Es besteht weitgehend Konsens darüber, dass die psychischen Belastungen mit dem Wandel der Arbeitswelt zunehmen. Schon vor mehr als zwei Jahrzehnten konstatierte Mergner (1989) angesichts von Veränderungen im "gesellschaftlichen System der Arbeit“ einen „Bedeutungsgewinn" psychischer Belastungen. Gegenwärtig werden verschiedene, in die genannte Richtung wirkende Entwicklungstendenzen ausgemacht (vgl. hierzu etwa: Pröll/Gude 2003):

- eine fortlaufende Beschleunigung von Fertigungs-, Dienstleistungs- und Kommunikationsprozessen aufgrund globalen Wettbewerbs, technischen Fortschritts und anhaltender Rationalisierungsdynamik; - zunehmende geistige Arbeit und steigende Anforderungen an Qualifikation und beständige Weiterbildung (Tertiarisierung, Trend zur Informations- und Wissensgesellschaft);

- verstärkter Einsatz neuer Technologien, die permanente Erreichbarkeit ermöglichen sowie Multitasking und die Entgrenzung der Arbeit fördern (Work Extension Technologies);

- erhöhte Eigenverantwortung der Beschäftigten für den gelungenen Ablauf und Erfolg ihrer Arbeit in Verbindung mit steigender Komplexität der Arbeitsanforderungen und neuen Steuerungssystemen (Subjektivierung von Arbeit);

- diskontinuierlichere Beschäftigungsverhältnisse, steigende Mobilitätsanforderungen und berufliche Unsicherheit sowie flexible Personaleinsatzkonzepte im Kontext permanenter Restrukturierungsprozesse;
- zunehmende Instabilität sozialer Beziehungen in Zusammenhang mit Tätigkeits- und Berufswechsel und wachsender Konkurrenz am Arbeitsplatz.

Die Entwicklungen der Arbeit sind hier nur kursorisch und verallgemeinernd skizziert. In diesem Artikel soll aufgezeigt werden, welchen Stellenwert psychische Belastungen mittlerweile einnehmen, welche Belastungsschwerpunkte sich herauskristallisieren, wie diese wahrgenommen werden und welche Herausforderungen sich daraus - implizit - auch für die zukünftige Gestaltung der Erwerbsarbeit ergeben. Abschnitt 2 beginnt daher mit einer Darstellung der Arbeitsbedingungen, ihrer Veränderungen im Zeitverlauf und der jeweiligen Wahrnehmung einzelner Belastungen durch die Beschäftigten auf repräsentativer Basis. In Abschnitt 3 geht es um die Frage, inwieweit psychische Belastungen mittlerweile in Unternehmen und auf überbetrieblicher Akteursebene thematisiert und (angemessen) bearbeitet werden. Dazu werden Ergebnisse aktueller Erhebungen im europäischen Kontext vorgestellt. Abschließend werden einige Handlungsoptionen für die Arbeitspolitik und für die Forschung aufgezeigt (Abschnitt 4).

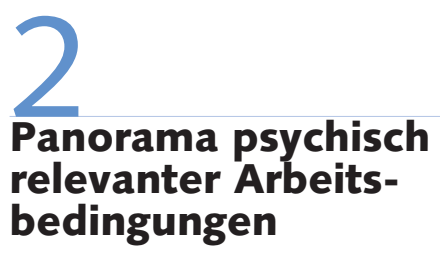

Um empirische Informationen über die Verbreitung, Verteilung und Entwicklung von Arbeitsbelastungen in der erwerbstätigen Bevölkerung zu erhalten, muss auf repräsentative Befragungen zurückgegriffen werden. Für Deutschland können folgende Untersuchungen als die wichtigsten Datenquellen gelten:

- Die vom Bundesinstitut für Berufsbildung (BIBB) zusammen mit dem Institut für Arbeitsmarkt- und Berufsforschung (IAB) bzw. der Bundesanstalt für Arbeitsschutz und Arbeitsmedizin (BAuA) alle sechs bis sieben Jahre durchgeführten Erwerbstätigenbefragungen (Stichprobengröße 2005/06: $\mathrm{N}=20.000$ ).

- Die seit 1991 alle fünf Jahre durchgeführten European Working Conditions Surveys der Europäischen Stiftung zur Verbesserung der Lebens- und Arbeitsbe- dingungen, Dublin (deutsche Teilstichprobe im Jahr 2005: $\mathrm{N}=1018$ ).

- Die seit 2007 im ersten Quartal eines jeden Jahres stattfindenden Befragungen abhängig Beschäftigter zum DGB-Index Gute Arbeit (2009: N = 7930). ${ }^{1}$

Im Überblick der Befragungsergebnisse zeigt sich, dass psychisch relevante Belastungen das Spektrum der Arbeitsbedingungen inzwischen deutlich bestimmen (Abbildungen 1-4). Dennoch bleibt die Verbreitung körperlicher Belastungen beachtlich, wie die ermittelten Betroffenheitsquoten, etwa für das Handhaben schwerer Lasten (20-23\%), repetitive Bewegungen (41\%) oder Lärm (20-24\%), erkennen lassen. Bei einzelnen Belastungsarten (z. B. Lärm) gibt es sogar Hinweise auf einen mittel- oder langfristigen (Wieder-)Anstieg. Wenn nun in den folgenden Ausführungen die psychischen Aspekte der Arbeitsbedingungen im Mittelpunkt stehen und auf die Erörterung der körperlichen Belastungen weitgehend verzichtet wird, ist also keineswegs unterstellt, Letztere seien nur mehr von marginaler Bedeutung oder generell auf dem Rückzug.

\subsection{INTENSITÄT DER ARBEIT}

Die hohe Intensität der Leistungsverausgabung nimmt nach allen hier herangezogenen Erhebungen eine zentrale Stellung in der Bewertung der Arbeitsbedingungen durch die Beschäftigten ein. So gaben in der BIBB/BAuA-Erwerbstätigenbefragung 2005/06 53,5 \% der Befragten an, starker Termin- und Leistungsdruck komme bei ihrer Arbeit „häufig“ vor, was im Ver-

\footnotetext{
Die in diesem Aufsatz referierten Daten der BIBB/ BAuA- bzw. BIBB/IAB-Erwerbstätigenbefragungen sind folgenden Veröffentlichungen entnommen: BIBB (2008); Beermann et al. (2007); Jansen (2000). Als Quellen von EWCS-Daten wurden herangezogen: Paoli $(1992,1997)$ sowie European Foundation (2007, 2008). Bei den zitierten Ergebnissen aus den DGB-Index-Befragungen handelt es sich, sofern nicht anders angegeben, um bislang unveröffentlichte Daten, die den Autoren zur Verfügung gestellt wurden. - Bei der Zusammenschau der Ergebnisse aus den vorgenannten Untersuchungen ist zu berücksichtigen, dass diese sich hinsichtlich der Erhebungstechnik, des Fragebogenaufbaus, der Itemformulierungen, der Erhebungszeitpunkte und des Befragungskontextes zum Teil deutlich unterscheiden. Das spricht nicht generell gegen die Möglichkeit, die Resultate sinnvoll zueinander in Beziehung zu setzen, muss aber als Aufforderung verstanden werden, hierbei sensibel und kritisch vorzugehen.
} 

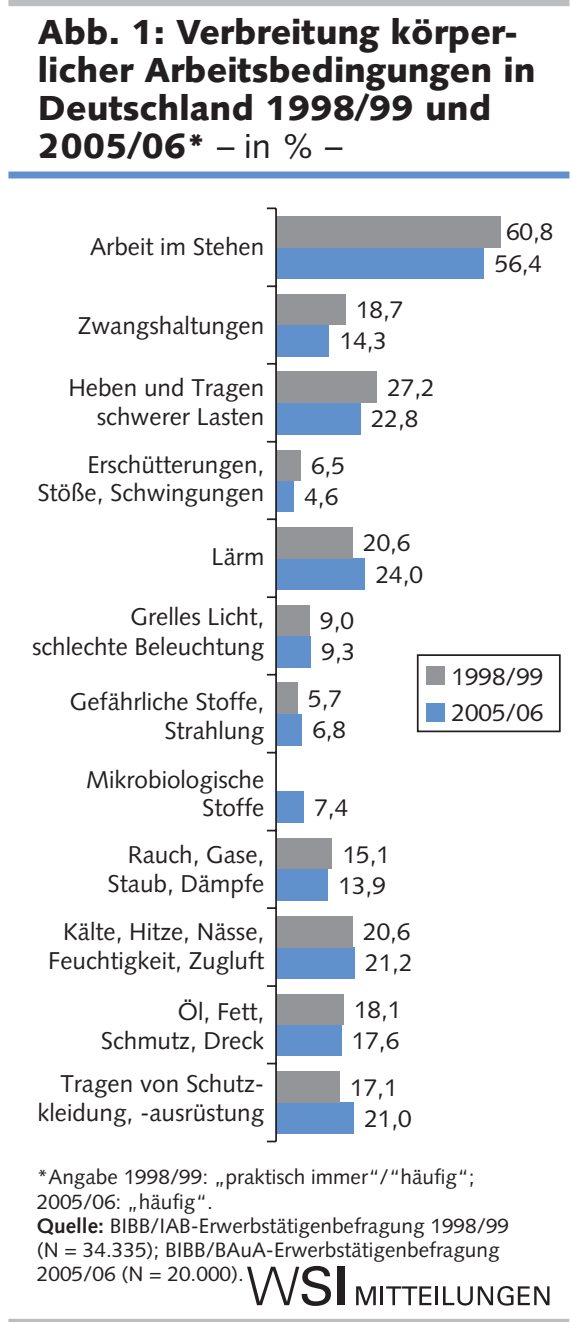

gleich mit früheren BIBB/IAB-Befragungen (1998/99: 50\%; 1985: $44 \%$ ) auf eine langfristige Zunahme hindeutet. 2005/06 bestätigten zudem 44,0 \% der Erwerbstätigen, dass sie häufig sehr schnell arbeiten müssten. Darüber hinaus wurde die erstmals gestellte Zusatzfrage, ob man sich durch das Vorhandensein bestimmter Arbeitsbedingungen auch belastet fühle, im Falle starken Termin- und Leistungsdrucks sowie sehr schnellen Arbeitens von $52 \%$ bzw. $36 \%$ der Betroffenen (umgerechnet 17,0 bzw. 10,7 Mio. Erwerbstätige) bejaht. Ein ähnliches Bild vermitteln die European Working Conditions Surveys (EWCS), denen zufolge der Anteil der deutschen Erwerbstätigen, die mindestens zur Hälfte ihrer Arbeitszeit unter Termindruck arbeiten, seit 1991 kontinuierlich von $46 \%$ bis auf $55 \%$ gestiegen ist. Von einem überwiegend sehr hohen Arbeitstempo berichteten hier sogar $58 \%$ der Befragten - rund 15 Prozentpunkte mehr als in den drei vorangegangenen Surveys.

Aktuellere Daten zur Einschätzung der Arbeitsintensität durch die Erwerbstätigen

\section{Abb. 2: Verbreitung psychischer \\ Arbeitsanforderungen in Deutschland 1998/99 und 2005/06* - in \% -}

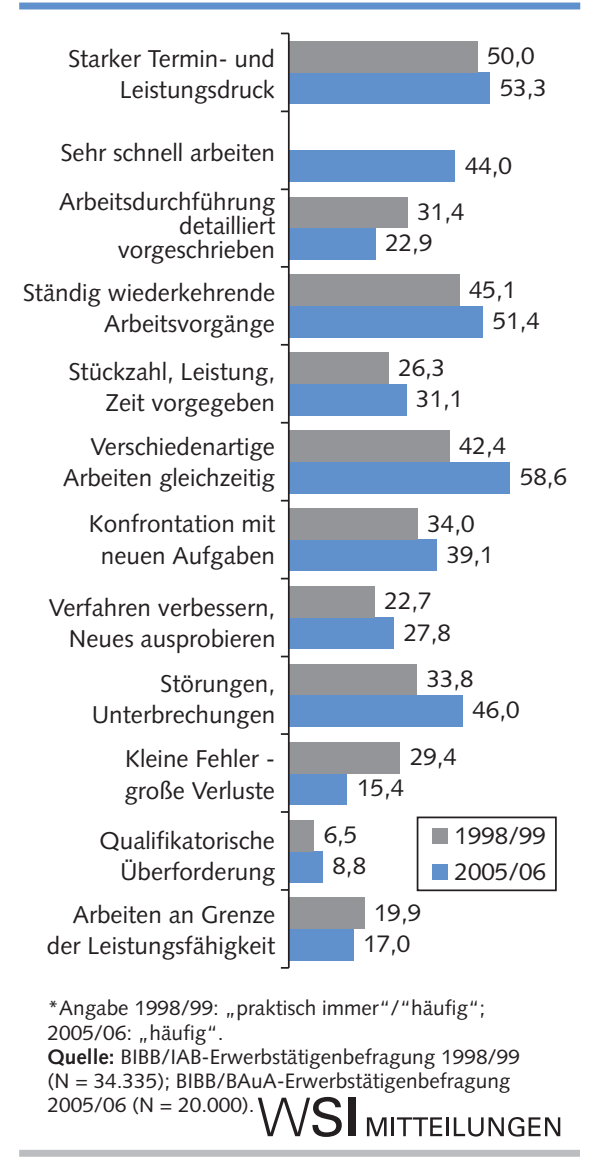

Abb. 3: Psychische Belastungen bei der Arbeit unter deutschen Erwerbstätigen 1991 bis 2005 - in \% -

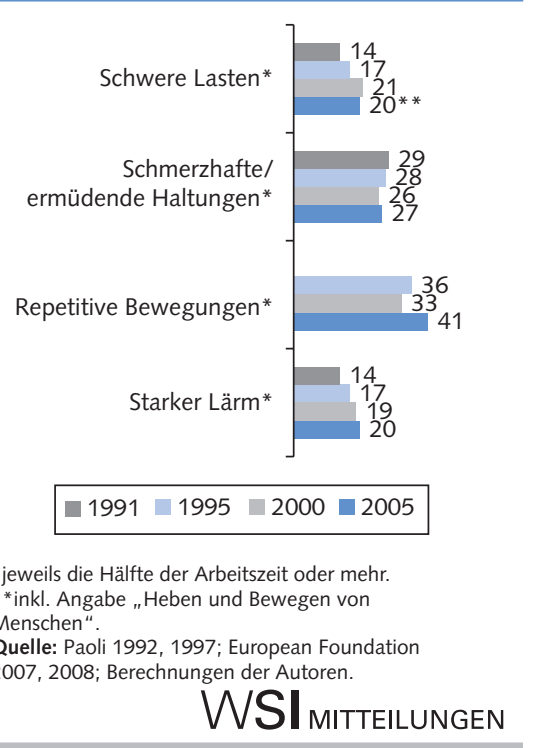

liefern die Befragungen zum DGB-Index Gute Arbeit. Der Prozentsatz derer, die zuletzt (2009) angaben, in hohem bis sehr
Abb. 4: Psychische Belastungen und Handlungsspielräume bei der Arbeit unter deutschen Erwerbstätigen 1991 bis 2005 - in \% -

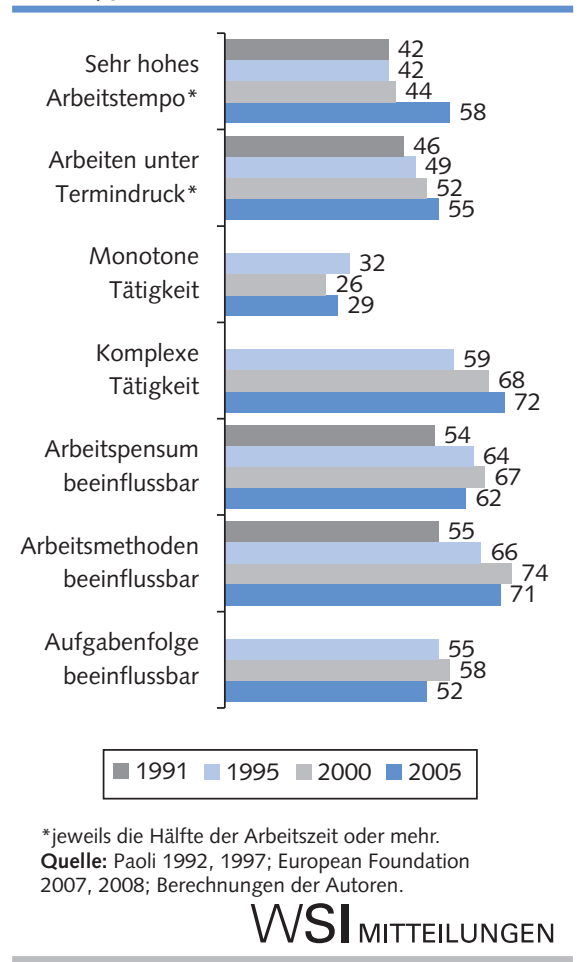

hohem Maße sich „in der Arbeit gehetzt zu fühlen“ und „Zeitdruck zu haben“, fällt zwar - wohl wegen der „zugespitzteren“, bereits unmittelbar auf das subjektive Belastungsempfinden abstellenden Frageformulierung - niedriger aus als die oben referierten $\mathrm{BIBB} / \mathrm{BAuA}$ - und EWCS-Werte, ist mit $36 \%$ aber dennoch beträchtlich.

Häufig in Zusammenhang mit hohem Zeitdruck sowie wachsender Komplexität und enger werdender Markt- und Kundenbindung von Arbeitsprozessen steht das Auftreten von Störungen und Unterbrechungen bei der Arbeit: Hiermit sehen sich laut BIBB/BAuA-Befragung 2005/06 $46 \%$ der Erwerbstätigen (rund 12 Prozentpunkte mehr als 1998/99) häufig konfrontiert, von denen wiederum fast $60 \%$ angeben, dies belaste sie.

\subsection{LÄNGE UND LAGE DER ARBEITSZEIT}

Zumindest bei den westdeutschen Vollzeitbeschäftigten ist seit Mitte der 1990er Jahre - nach langwährender Abwärtsentwicklung - eine Tendenz zur Ausweitung der durchschnittlichen effektiven Wochenarbeitszeit wirksam (Lehndorff et al. 2009). $26 \%$ aller Vollzeitarbeitnehmer und 
-arbeitnehmerinnen gaben laut Erhebung zum DGB-Index 2009 an, wöchentlich 46 Stunden und mehr zu arbeiten (Lenhardt et al. 2010, S. 471). Darüber hinaus nehmen "atypische" und wechselnde Arbeitszeitlagen zu. So sind während der vergangenen beiden Jahrzehnte (1991-2008) die Anteile der Beschäftigten, die an Samstagen und Sonntagen arbeiten, deutlich gestiegen (von $33 \%$ auf $45 \%$ bzw. von $17 \%$ auf $26 \%$ ), und auch die Nacht- und die Wechselschichtarbeit hat sich - wenngleich in geringerem Maße - ausgeweitet (von $13 \%$ auf $16 \%$ bzw. von $13 \%$ auf $17 \%$ ) (HBS 2009, S. 2). In der DGB-Index-Befragung 2009 gab ein knappes Drittel der Beschäftigten an, zu versetzten oder gar völlig unregelmäßigen Zeiten zu arbeiten (Lenhardt et al. 2010, S. 471), außerdem sehen 40 \% ihre Bedürfnisse bei der Planung der Arbeitszeit nicht oder nur in geringem Maße berücksichtigt und $60 \%$ betrachten ihren Einfluss auf die eigene Arbeitszeitgestaltung als bestenfalls geringfügig (ebd., S. 473). Von langen Arbeitszeiten, Schicht- und Nachtarbeit sowie stark variierender Lage und Dauer der (täglichen) Arbeitszeit ausgehende negative gesundheitliche Effekte sind empirisch vielfältig belegt (Wirtz et al. 2009; Beermann 2010; Janßen/Nachreiner 2004).

\subsection{INHALTLICHE ANFORDERUNGEN DER ARBEIT}

Ausgeprägte Diversität, Komplexität und Wandelbarkeit der Arbeitsaufgaben sind für große (und tendenziell gewachsene) Teile der Beschäftigten weitere zentrale Merkmale ihrer beruflichen Tätigkeit. Knapp 59 \% der in der BIBB/BAuA-Erhebung Befragten - über 16 Prozentpunkte mehr als 1998/99 - gaben an, häufig verschiedenartige Arbeitsvorgänge gleichzeitig im Auge haben zu müssen. In ebenfalls gestiegenem Umfang wurde 2005/06 davon berichtet, dass man bei der Arbeit häufig mit neuen Aufgaben konfrontiert sei $(39,1 \%$ gegenüber $34,0 \%$ in $1998 / 99)$ oder häufig Verfahren verbessere und Neues ausprobiere (27,8 \% gegenüber 22,7 \%). Eine stimmige Ergänzung dieser Befunde bildet der von $59 \%$ (1995) auf $72 \%$ (2005) gewachsene Anteil derer, die in den deutschen EWCS-Teilstichproben angaben, ihre Berufstätigkeit beinhalte komplexe Arbeitsaufgaben. Auch die Einschätzung, bei der Arbeit neue Dinge zu lernen, ist mit $66 \%$ weit verbreitet (wenngleich nicht mehr ganz so häufig vorhanden wie in den EWCS-Befragungen 1995 und 2000). Auf die ähnlich gelagerte Frage, ob die Arbeit es ermögliche, eigenes Wissen und Können weiterzuentwickeln, antworteten in der DGB-Index-Befragung 200857 \% der Beschäftigten, dies sei in hohem oder sehr hohem Maße der Fall (Fuchs 2009, S. 70).

Grundsätzlich stellen diese Ergebnisse eine gute Nachricht dar, insofern sie einen Trend hin zu vielfältigeren, abwechslungsreicheren und anspruchsvolleren Aufgabenzuschnitten signalisieren. Allerdings kann aus erhöhter Anforderungsvielfalt und -komplexität ein Bewältigungsproblem für die Beschäftigten erwachsen, wenn sie z.B. mit unzureichender Qualifizierung, häufigen Störungen oder starkem Zeitdruck einhergehen. Hier dürften wesentliche Gründe dafür liegen, dass $12 \%$ derjenigen, die sich in ihrer Arbeit gelegentlich oder häufig vor neue Aufgaben gestellt sehen (hochgerechnet 3,7 Mio. Erwerbstätige), dies nicht (nur) als bereichernd, sondern (auch) als belastend empfinden.

Außerdem sollte nicht aus dem Blick geraten, dass eintönige, gleichförmige Tätigkeiten nach wie vor den Arbeitsalltag sehr vieler Beschäftigter prägen. Laut EWCS 2005 betrachteten immerhin $29 \%$ der deutschen Befragten - etwas weniger als 1995, aber mehr als im Jahr 2000 - Monotonie als ein Merkmal ihrer Tätigkeit. Von kurzzyklisch (nach weniger als einer Minute) wiederkehrenden Arbeitsaufgaben berichtete ein seit 2000 nahezu unveränderter Anteil von knapp $22 \%$. Rund $33 \%$ gaben an, in ihrer Arbeit nichts Neues dazuzulernen (Zunahme seit 1995). Hochgerechnet 3,6 Mio. Menschen empfinden gemäß BIBB/BAuA-Erhebung 2005/2006 die Erfahrung detailliert sich wiederholender Arbeitsgänge subjektiv auch als belastend.

\subsection{HANDLUNGSSPIELRAUM, TRANSPARENZ UND SOZIALE UNTERSTÜTZUNG}

Arbeitsbezogene Handlungs- und Entscheidungsspielräume können als Ressourcen begriffen werden, die Gesundheit und Wohlbefinden der Beschäftigten stützen und fördern, indem sie die Bewältigung von Arbeitsanforderungen erleichtern, persönliche Entfaltungschancen eröffnen und eine positive Qualität des Arbeitserlebens erzeugen. In der BIBB/BAuA-

\section{Abb. 5: Verbreitung arbeits- bezogener psychosozialer Ressourcen in Deutschland \\ 2005/06* - in \% -}

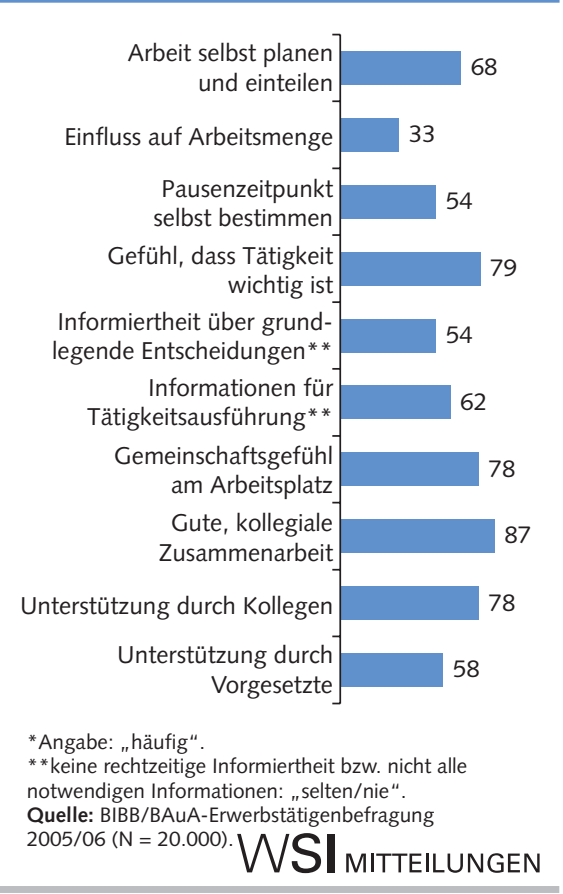

Erhebung 2005/06 trafen nur mehr 22,9\% der Befragten die Aussage, dass ihnen die Arbeitsdurchführung häufig bis in alle Einzelheiten vorgeschrieben sei - sieben Jahre zuvor war es noch ein knappes Drittel. Quasi spiegelbildlich dazu verhält sich der Befund, dass gut zwei Drittel der Erwerbstätigen nach eigener Auskunft ihre Arbeit überwiegend selbst planen und einteilen können. Während immerhin noch $54 \%$ der Beschäftigten den Zeitpunkt ihrer Pausen häufig selbst bestimmen, liegt die Definition des zu leistenden Arbeitspensums größtenteils außerhalb ihrer Einflussmöglichkeiten: Gerade einmal $33 \%$ berichten von einer mehr als nur gelegentlichen Beeinflussbarkeit der ihnen zugewiesenen Arbeitsmenge (Abbildung 5).

Im EWCS 2005 gaben $71 \%$ der Befragten an, über die von ihnen anzuwendenden Arbeitsmethoden entscheiden zu können, $52 \%$ bestätigten dies im Hinblick auf die Reihenfolge der zu bearbeitenden Aufgaben. Im Unterschied zur BIBB/BAuAErhebung berichtete hier eine deutliche Mehrheit auch von der Beeinflussbarkeit des Arbeitstempos/-pensums. In längerfristiger Betrachtung sprechen die EWCSDaten zudem für einen tendenziellen Autonomiezuwachs hinsichtlich der Wahl der Arbeitsmethoden und der Regulierung des Arbeitstempos/Leistungspensums, aller- 
dings scheint dieser Trend seit der Jahrtausendwende gebrochen. In dieses Bild fügt sich wiederum ein weiterer Befund der BIBB/BAuA-Befragung 2005/06: Hiernach berichtet ein seit Ende der 1990er Jahre gewachsener Teil der Erwerbstätigen (31 \% gegenüber $26 \%$ ), Stückzahlen, Mindestleistungen oder für deren Erbringung zur Verfügung stehende Zeitrahmen seien genau vorgeschrieben. Während also die Vorgabe der konkreten Tätigkeitsvollzüge an Rigidität verloren hat, scheint Letzteres im Hinblick auf die Ergebnis- und Zeitvorgaben keineswegs der Fall zu sein.

Die Fähigkeit, die Arbeitstätigkeit effektiv selbst regulieren zu können, hängt maßgeblich von der Verfügbarkeit diesbezüglich relevanter Informationen ab. Was das betrifft, haben die Beschäftigten laut BIBB/BAuA-Befragung eine insgesamt recht positive Einschätzung: $62 \%$ von ihnen gaben hier an, (fast) immer alle für eine ordentliche Tätigkeitsausführung notwendigen Informationen zu erhalten. Andererseits verweist ein Befragtenanteil von $37 \%$, bei dem es hin und wieder oder öfter zu einem die Ausführung der Arbeitstätigkeit beeinträchtigenden Informationsmangel kommt, auf einen durchaus ernstzunehmenden Handlungsbedarf, zumal selbst ein nur gelegentliches Auftreten dieses Problems in erheblichem Umfang ( $\mathrm{zu}$ $73 \%$ ) als belastend erlebt wird. Bedeutsam für die Beschäftigten sind indessen nicht nur die die unmittelbare Arbeitsausführung betreffenden Informationen, sondern auch solche, die Transparenz hinsichtlich der betrieblichen Situation und Entwicklungsperspektive herzustellen vermögen. Hier liefert die BIBB/BAuA-Erhebung einen nicht ganz so günstigen Befund: Zwar sagen die Befragten mehrheitlich (54\%), sie würden in aller Regel rechtzeitig über einschneidende Entscheidungen, Veränderungen und Zukunftspläne informiert, der Anteil derer, bei denen dies zumindest manchmal auch nicht der Fall ist, liegt mit gut $45 \%$ aber doch auf einem vergleichsweise hohen Niveau.

Auch der sozialen Unterstützung am Arbeitsplatz kommt die Funktion einer Gesundheitsressource im o.g. Sinne zu. Entgegen der oft geäußerten Annahme ausgeprägten Konkurrenzverhaltens innerhalb der Belegschaften, grassierenden Mobbings u.ä. werden die Beziehungen zu den Kollegen im Allgemeinen offenbar nach wie vor sehr positiv bewertet. Laut BIBB/BAuA-Befragung 2005/06 sehen große Teile der Beschäftigten die kollegialen Beziehungen durch gute Kooperation (87\%), engen Zusammenhalt („Gemeinschaftsgefühl“) (78\%) und gegenseitige Unterstützung (78 \%; EWCS 2005: 65 \%) gekennzeichnet. Verglichen damit fällt das Urteil über die Vorgesetzten verhaltener, aber immer noch recht günstig aus: $58 \%$ (ebenso viele wie im EWCS 2005) attestieren diesen ein in der Regel unterstützendes Verhalten.

Von der Ressourcenseite her betrachtet scheint es um die Arbeitsbedingungen hierzulande also gar nicht einmal so schlecht bestellt zu sein. Als Entwarnung bezüglich der aktuellen und künftigen Bedeutung psychischer Belastungsprobleme bei der Arbeit ist diese Einschätzung gleichwohl nicht zu verstehen, und zwar aus mehreren Gründen:

(1) Was das Ressourcenpotenzial der Beschäftigten betrifft, weisen empirische Daten auf partielle Rückschritte bzw. Stagnationstendenzen während des vergangenen Jahrzehnts sowohl in Deutschland als auch in anderen europäischen Ländern - etwa Frankreich (Bué et al. 2007) oder Dänemark (Pejtersen/Kristensen 2009) - hin.

(2) Dänischen Untersuchungen zufolge hat der gesundheitlich protektive Einfluss arbeitsbezogener Handlungs- und Entscheidungsspielräume - möglicherweise aufgrund eines „Bedeutungswandels“, den diese erfahren haben - seit Anfang der 1990er Jahre kontinuierlich abgenommen (Albertsen/Burr 2009). Der erwähnte Bedeutungswandel könnte in einer immer deutlicher zutage tretenden Ambivalenz von Autonomie und Selbststeuerung - Ausdrucksformen eines Humanisierungsfortschritts und Durchsetzungsmodi forcierter Leistungsverausgabung - bestehen.

(3) Intensivierungs- und flexibilisierungsbedingte Anforderungen in der Arbeit drohen zunehmend auf die außerberufliche Lebenssphäre überzugreifen und deren Funktion als Raum, der innere Distanzierung vom Berufsalltag, Entspannung, Erholung und die Realisierung sozialer Bedürfnisse ermöglicht, zu unterminieren: Laut DGB-Index-Befragung 2007 sind jeweils 42 \% der Beschäftigten der Auffassung, dass das Verhältnis zwischen Beruf und Privatleben bei ihnen nicht oder nur wenig ausgewogen sei bzw. dass ihre Arbeit kaum genügend Zeit für Familie, Freundschaften und private Interessen lasse (DGB 2008, S. 4f.).

Das Gesamtbild psychischer Belastungen und ihrer Bewältigungsmöglichkeiten bliebe unvollständig, wenn man die Tatsache außer Acht ließe, dass Anfang 2008 (also vor Ausbruch der globalen Wirtschafts- und Finanzkrise) nicht weniger als $34 \%$ der Beschäftigten von subjektiv stark bis sehr stark belastenden Ängsten um ihre berufliche Zukunft berichteten (Fuchs 2009, S. 71). Angesichts dessen spricht einiges dafür, dass langfristig hohe Arbeitslosenzahlen, zunehmende prekäre Beschäftigungsverhältnisse, häufige betriebliche Restrukturierungen und rascher veraltende arbeitsbezogene Wissensbestände zu einem tendenziellen Schwinden von - realer und "gefühlter“ Sicherheit führen.

\section{3 \\ Umgang mit dem Problem psychischer Belastung}

$\mathrm{Ob}$ und inwiefern Erkenntnisse zu psychischen Belastungen in der Arbeitswelt handlungsrelevant werden, hängt nicht zuletzt von der Sensibilisierung der Arbeitsschutzakteure für dieses Thema und ihren darauf bezogenen Sichtweisen ab auf der politisch-institutionellen wie auf der betrieblichen Ebene. Hierzu lagen bislang aber nur wenige Daten vor.

Aktuelle empirische Hinweise auf den Umgang der politisch-institutionellen und der betrieblichen Akteure mit dem Thema arbeitsbezogener psychosozialer Risiken liefern Daten aus zwei neueren europäischen Forschungsprojekten, deren Ergebnisse an anderer Stelle ausführlich dokumentiert sind (Ertel/Stilijanow 2009). Es handelt sich zum einen um das Projekt PRIMA-EF (Psychosocial Risk Management-European Framework), das darauf abzielte, vor dem Hintergrund des europäischen Integrationsprozesses einen abgestimmten Handlungsrahmen für die Bewältigung von psychosozialen Risiken bei der Arbeit zu entwickeln. Dargestellt werden ausgewählte Ergebnisse einer EUweiten Befragung von institutionellen Arbeitsschutzakteuren (Repräsentanten staatlicher Einrichtungen sowie Vertreter von Unternehmerverbänden und Gewerk- 
schaften; $\mathrm{N}=75$ ), die im Rahmen dieses Projektes durchgeführt wurde.

Was die betriebliche Handlungsebene betrifft, werden zum anderen Ergebnisse einer im Jahr 2009 durchgeführten repräsentativen Befragung von betrieblichen Arbeitsschutzakteuren in 30 europäischen Ländern herangezogen. Diese von der Europäischen Agentur für Sicherheit und Gesundheitsschutz am Arbeitsplatz in Auftrag gegebene Unternehmensbefragung (ESENER - European Survey of Enterprises on New and Emerging Risks) erhob von den verantwortlichen Akteuren des Managements $(\mathrm{N}=28.649)$ und der betrieblichen Interessenvertretung $(\mathrm{N}=7.226)$ detaillierte Informationen darüber, wie insbesondere psychosoziale Risiken am Arbeitsplatz thematisiert und praktisch angegangen werden. Die in diesem Artikel referierten Ergebnisse beziehen sich ausschließlich auf die deutsche Teilstichprobe $(\mathrm{N}=1510)$.

\subsection{POLITISCH-INSTITUTIONELLE HANDLUNGSEBENE}

Die im Rahmen von PRIMA-EF durchgeführte Befragung unter Stakeholdern aus den Bereichen Staat, Gewerkschaften und Arbeitgeberorganisationen zielte in erster Linie darauf ab, das Problembewusstsein und somit die Handlungsbereitschaft dieser Zielgruppen auf dem Gebiet des Arbeitsschutzes, speziell der Stressprävention, zu ermitteln, wobei besonderes Augenmerk auf Gemeinsamkeiten und Unterschiede in der Problemwahrnehmung zwischen den verschiedenen Akteurgruppen einerseits sowie zwischen den Vertretern der 15 „alten“ und denen der zwölf „neuen“ EU-Staaten andererseits gerichtet war.

Der PRIMA-EF-Befragung nach zu urteilen, besteht an der grundsätzlichen Bedeutung von psychosozialen Risiken und Stress für die arbeitsschutzpolitischen Schlüsselakteure in Europa ganz überwiegend kein Zweifel: 71 \% von ihnen betrachten diese Problematiken als „wichtige Anliegen für den Arbeitsschutz in ihrem Land“. Dabei zeigt sich eine deutlich geringere Zustimmungsquote der Befragten aus den neuen EU-Ländern (59\%), was sich mit anderen Problemgewichtungen, schwächer ausgebildeten Strukturen und geringeren Ressourcen im dortigen Arbeitsschutz in Verbindung bringen lässt. Noch ausgeprägter sind die Antwortdifferenzen zwischen den verschiedenen Akteurgrup-

Tabelle 1: Bedeutsamkeit verschiedener psychosozialer Risiken im eigenen Betrieb* - in \% -

\begin{tabular}{|c|c|c|}
\hline & $\begin{array}{l}\text { Management- } \\
\text { vertreter }\end{array}$ & $\begin{array}{l}\text { Arbeitnehmer- } \\
\text { vertreter }\end{array}$ \\
\hline Zeitdruck & 71,7 & 79,5 \\
\hline Schlechte Kommunikation zw. Management und Beschäftigten & 32,4 & 49,4 \\
\hline Schlechte Kooperation unter den Kollegen & 26,0 & 29,3 \\
\hline $\begin{array}{l}\text { Fehlende Kontrolle der Beschäftigten über die Organisation } \\
\text { ihrer Arbeit }\end{array}$ & 18.0 & 23,3 \\
\hline Arbeitsplatzunsicherheit & 26,0 & 33,7 \\
\hline Umgang mit schwierigen Kunden, Patienten, Schülern etc. & 47,7 & 47,8 \\
\hline Probleme im Verhältnis zwischen Mitarbeitern und Vorgesetzten & 28,0 & 46,2 \\
\hline Lange oder unregelmäßige Arbeitszeiten & 28,0 & 53,0 \\
\hline Eine unklare Personalpolitik & 14,6 & 39,4 \\
\hline $\begin{array}{l}\text { Diskriminierung (z.B. aufgrund von Geschlecht, Alter oder } \\
\text { ethnischer Herkunft) }\end{array}$ & 4,4 & 6,0 \\
\hline \multirow{2}{*}{\multicolumn{3}{|c|}{$\begin{array}{l}\text { * "Ja"-Antworten, Mehrfachnennungen möglich; Frageformulierung: „Verschiedene Faktoren können zu Stress, Gewalt } \\
\text { und Belästigung am Arbeitsplatz beitragen; sie betreffen die Art, wie Arbeit organisiert wird, und werden oft ,psychosoziale } \\
\text { Risiken' genannt. Bitte sagen Sie mir für jedes der folgenden psychosozialen Risiken, } \\
\text { ob dies ein Anliegen in Ihrem Betrieb ist." }\end{array}$}} \\
\hline & & ITTEILUNGEN \\
\hline
\end{tabular}

pen, besonders zwischen den Vertretern der Arbeitgeberverbände (50\%) und denen der Gewerkschaften ( $85 \%)$. Letzteres lässt sich als Ausdruck unterschiedlicher Interessenlagen und Handlungsprioritäten im Arbeitsschutz deuten (Natali et al. 2008).

Die konkretisierende Frage, ob in ihrem Land Probleme in Verbindung mit Stress am Arbeitsplatz ihrer „Bedeutung entsprechend anerkannt“ werden, wird von den Arbeitsschutzakteuren zu einem deutlich geringeren Anteil (30\%) bejaht, wobei sich auch hier markante Unterschiede zwischen den Vertretern der 15 „alten“ (43\% Zustimmung) und denen der zwölf „neuen“EU-Länder (13 \%) einerseits sowie zwischen den Vertretern von Arbeitgeberverbänden (50\%) und Gewerkschaftsvertretern $(15 \%)$ andererseits ergeben. Bei aller (nicht zuletzt der geringen Stichprobengröße geschuldeten) Vorsicht lassen sich die referierten Befunde dahingehend interpretieren, dass ein Großteil der arbeitsschutzpolitischen Schlüsselakteure inzwischen wohl von der hohen Relevanz der Stressproblematik überzeugt ist, zugleich aber die Aufmerksamkeit, die dieser Problematik in ihrem jeweiligen Land tatsächlich entgegengebracht wird, als inadäquat ansieht. Damit ist implizit auch die betriebliche Ebene angesprochen, auf der der Umgang mit arbeitsbedingten psychischen Belastungen real stattfindet.

\subsection{BETRIEBLICHE HANDLUNGSEBENE}

Im ESENER-Survey wurden Angehörige des Managements und der Arbeitnehmervertretung in gleicher Weise danach gefragt, ob verschiedene in einer Liste aufgeführte psychosoziale Risikofaktoren in ihrem Betrieb von Belang sind. Hierbei zeigte sich eine in beiden Gruppen gleichermaßen überragende Bedeutungseinschätzung des Faktors "Zeitdruck“ wie auch eine übereinstimmend als hoch angesehene Relevanz des Faktors „Umgang mit schwierigen Kunden“. Anderen Problemen - etwa schlechter Kommunikation zwischen Management und Beschäftigten, Schwierigkeiten im Mitarbeiter-Vorgesetzten-Verhältnis, einer unklaren Personalpolitik oder langen/unregelmäßigen Arbeitszeiten - wird hingegen eine sehr unterschiedliche betriebliche Relevanz beigemessen (Tabelle 1).

Im Vergleich zu anderen Themen des Arbeits- und Gesundheitsschutzes schätzte jeweils die relative Mehrheit der befragten Management- und Arbeitnehmervertreter (53,6 \% bzw. 49,4 \%) den Umgang mit psychosozialen Risiken als schwieriger ein, deutlich weniger (33,0 \% bzw. 38,8 \%) sehen diesbezüglich keinen Unterschied, und nur eine kleine Minderheit (7,5 \% bzw. 9,2 \%) hält das Problem psychosozialer Risiken für leichter handhabbar. Bei den Gründen, die nach Auffassung der Managementvertreter den Umgang mit psychosozialen Risiken im Betrieb „besonders schwierig“ machen, fällt neben Ressourcendefiziten (Zeit, Personal, Geld) (Nennungshäufigkeit 54,5\%), fehlender Ausbildung bzw. fehlendem Fachwissen (54,1 \%) und mangelndem Problembewusstsein (55,6 \%) der mit $65 \%$ besonders hohe Anteil derer auf, die auf den „heiklen“ Charakter des Themas hinweisen. 


\section{Abb. 6: Gründe für den eigenen Betrieb, sich mit psychosozialen Risiken zu befassen* - in \% -}

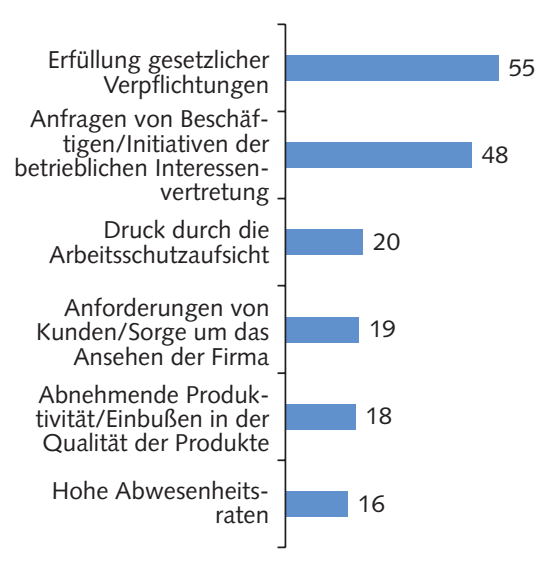

*Angaben von Managementvertretern aus deutschen Betrieben ( $N=1.510$; Mehrfachantworten möglich). Quelle: Survey ESENER 2009

\section{WSI MITTEILUNGEN}

$\mathrm{Ob}$ es an diesen „besonderen Schwierigkeiten“ liegt, dass nur $17 \%$ der Betriebe nach eigenen Angaben ein „Verfahren“ zum Umgang mit arbeitsbedingtem Stress etabliert haben, sei dahingestellt. Klar ist jedoch: Wenn ein solches Verfahren existiert, werden auch vermehrt konkrete Maßnahmen zur Risikominderung ergriffen, wie etwa Weiterbildungsangebote (86,6\% in Betrieben mit gegenüber 64,0\% der Betriebe ohne Verfahren), vertrauliche Beratung der Beschäftigten (82,8\% vs. 51,3\%), Einrichtung von Konfliktlösungsverfahren (65,5\% vs. $21,8 \%$ ) oder Veränderungen der Arbeitsorganisation (60,9\% vs. $42,6 \%)$.

Sofern nun Betriebe auf dem Gebiet psychosozialer Risiken tätig werden - was ausweislich der ermittelten Maßnahmehäufigkeiten in gar nicht mal so geringem Umfang der Fall zu sein scheint -, lohnt ein Blick auf die Gründe, aus denen sie sich hierzu veranlasst sehen. Im Vordergrund stehen hier laut ESENER-Survey (wohlgemerkt nach Angaben aus dem Management) nicht ökonomische bzw. marktbezogene Aspekte wie Kundenanforderungen, Fragen des Betriebsimages, Produktivitätsund Qualitätsprobleme oder hohe Fehlzeiten (an die anzuknüpfen häufig als der aussichtsreichste Weg zur Beförderung be- trieblicher Präventionspraxis gilt), sondern das Bestreben, gesetzliche Verpflichtungen zu erfüllen, sowie Anfragen und Initiativen seitens der Arbeitnehmer oder ihrer betrieblichen Vertretungen (Abbildung 6).

\section{Schlussfolgerungen}

Der eingangs postulierte Bedeutungszuwachs psychischer Belastungen im Kontext des Wandels der Arbeit wird durch die dargestellten repräsentativen Befragungsergebnisse bestätigt. Uneinheitlich stellt sich dagegen das Bild zur Entwicklung organisationaler Ressourcen dar: Einerseits ist ein langfristiger Zuwachs von Handlungs- und Entscheidungsspielräumen zu verzeichnen, der für verbesserte Möglichkeiten zur produktiven Bewältigung erhöhter Arbeitsanforderungen spricht, andererseits scheint genau diese Funktion zunehmend eingeschränkt.

Gleichzeitig machen die Daten zum Umgang politisch-institutioneller und betrieblicher Akteure mit der Thematik deutlich, dass der verbreiteten Einschätzung hoher Relevanz psychischer Belastungen für den Arbeitsschutz keine ausreichenden darauf bezogenen Handlungsorientierungen und -strategien gegenüberstehen. Wichtige Rahmenbedingungen zur Förderung entsprechender Aktivitäten, das haben die dargestellten Untersuchungsergebnisse ebenfalls gezeigt, stellen geregelte Verfahren und Gesetze dar. So leisten ganzheitliche Gefährdungsbeurteilungen gemäß Arbeitsschutzgesetz einen wichtigen Beitrag zur Analyse von Arbeitsbelastungen und zur Initiierung von Verbesserungsprozessen (vgl. den Beitrag von Satzer in diesem Heft).

Eine gesunde Gestaltung der Arbeit liegt in der Verantwortung und auch im Interesse der Unternehmen. Angesichts zunehmender "Selbststeuerung“ sind zugleich und verstärkt gesundheitsorientierte Einstellungen und Verhaltensweisen der Beschäftigten selbst gefordert, die im Prozess der Arbeit Gesundheitsrisiken vermeiden und gesundheitsförderliche Arbeits- bedingungen „nachfragen“ müssen, was gerade angesichts von Arbeitsplatzängsten und beruflicher Unsicherheit kein leichtes Unterfangen ist (Theorell 1997). Betriebliche Gesundheitsförderung muss, wenn sie wirksam und nachhaltig sein soll, zur ,gesamtbetrieblichen Aufgabe "werden, die an Langfristorientierungen des Managements im Sinne von „nachhaltigen Arbeitssystemen“ (Docherty et al. 2002) und an das Engagement betrieblicher Fach- und Führungskräfte ebenso wie an die Mitwirkung der Beschäftigten und ihrer Interessenvertretungen gebunden ist.

Dabei kann in Anbetracht der skizzierten Entwicklungen der Arbeit nicht mehr alleine auf Gestaltungskonzepte zurückgegriffen werden, die sich in der Vergangenheit bei restriktiven Tätigkeiten in der Produktion bewährt haben, wie beispielsweise „job enlargement" oder „job enrichment“. Vielmehr geht es heute - gerade mit Blick auf die wachsende Beschäftigtengruppe, deren Arbeit qualifiziert und eher „geistiger Natur" ist - auch um die Frage, wie angesichts hohen Leistungs- und Termindrucks drohende Überforderungen durch wachsende Aufgabenfülle und zunehmend „nach unten“ delegierte Ergebnisverantwortung vermieden werden können. Aus den dargestellten Belastungsproblemen ergeben sich sowohl eher traditionelle Gestaltungsaufgaben, wie die Umsetzung gesundheitszuträglicher Arbeitszeitregelungen, als auch die Anforderung, neue Handlungsansätze zu entwickeln, beispielsweise zur Vermeidung von Informationsüberflutung oder häufiger Störungen am Arbeitsplatz.

Wie konkrete Gestaltungskonzepte für jeweils spezifische Belastungskonstellationen aussehen können, wie individuelle und betriebliche Strategien wirksam ineinandergreifen und wie sie unterstützt und gefördert werden können, dies sind wichtige Forschungs- und Praxisfragen für die Zukunft. Um sie angemessen zu beantworten, bedarf es zugleich vertiefender Erkenntnisse und einer verbesserten Datengrundlage zu Ausprägungen, zur Entwicklung, zu Entstehungszusammenhängen und zu Folgen psychischer Belastungen in der Arbeitswelt. 
Albertsen, K./Burr, H. (2009): The prospective effect of control in work on changes in self-rated health from 1995-2005, http://www. arbejdsmiljoforskning.dk/upload/presentations/kal_210609.pdf (Zugriff: 20.04.2010)

Beermann, B. (2010): Nacht- und Schichtarbeit, in: Badura, B./Schröder, H./Klose, J./Macco, K. (Hrsg.): Fehlzeitenreport 2009. Arbeit und Psyche: Belastungen reduzieren - Wohlbefinden fördern, Berlin,

S. 71-82

Beermann, B./Brenscheidt, F./Siefer, A. (2007): Arbeitsbedingungen in Deutschland - Belastungen, Anforderungen und Gesundheit, in: Bundesanstalt für Arbeitsschutz und Arbeitsmedizin (Hrsg.): Gesundheitsschutz in Zahlen 2005, Dortmund/Berlin/Dresden, S. 28-45 Bundesinstitut für Berufsbildung (BIBB) (2008): BIBB/BAuA-Erwerbstätigenbefragung 2006 - Ergebnisse online, http://www.bibb.de/de/ 26901.htm (Zugriff: 15.02.2010)

Betriebskrankenkassen (BKK) Bundesverband (Hrsg.) (2008): BKK Gesundheitsreport 2008: Seelische Krankheiten prägen das Krankheitsgeschehen, Essen

Boes, A./Kämpf, T./Trinks, K. (2009): Eine neue Belastungskonstellation in der IT-Industrie - was tun? Vortrag auf dem Transferworkshop des Projekts DiWa-IT am 6. Oktober 2009 in München, http://www. diwa-it.de/img/content/091006_isf_transferworkshop_diwa.pdf (Zugriff 20.04.2010)

Bué, J./Coutrot, T./Hamon-Cholet, S./Vinck, L. (2007): Conditions de travail: une pause dans l'intensification du travail. Premières Synthèses DARES 01.2, Paris

DGB-Index Gute Arbeit GmbH (DGB) (Hrsg.) (2008): Work-Life-

Balance 2007 - Der Report. Wie die Beschäftigten die Vereinbarkeit von Berufs-, Familien- und Privatleben beurteilen, Berlin

Docherty, P./Forslin, J./(Rami) Shani, A.B. (Hrsg.) (2002): Creating

Sustainable Work Systems: Emerging Perspectives and Practice, London Ertel, M./Stilijanow, U. (2009): Bekämpfung psychosozialer Risiken am Arbeitsplatz - Politischer Kontext und Sozialer Dialog in der erweiterten EU, in: Arbeit 4, S. 353-365

European Foundation for the Improvement of Living and Working Conditions (2007): Third European Working Conditions survey: data, http://www.eurofound.europa.eu/ewco/3wc/3wcindex.htm (last updated 17 December 2007; Zugriff: 15.02.2010)

European Foundation for the Improvement of Living and Working Conditions (2008): Fourth European Working Conditions Survey - mapping the results, http://www.eurofound.europa.eu/ewco/ surveys/ewcs2005/results.htm (last updated: 23 July 2008; Zugriff: 15.02.2010)

Fuchs, T. (2009): Der DGB-Index, in: Bundesanstalt für Arbeitsschutz und Arbeitsmedizin (Hrsg.): Nutzerpotenziale von Beschäftigtenbefragungen, Dortmund/Berlin/Dresden, S. 47-85

Hasselhorn, H.M./Portuné, R. (2010): Stress, Arbeitsgestaltung und Gesundheit, in: Badura, B./Walter, U./Hehlmann, T. (Hrsg.): Betriebliche Gesundheitspolitik. Der Weg zur gesunden Organisation, Berlin, S. $361-376$
Hans-Böckler-Stiftung (HBS) (2009): Das gemeinsame Wochenende fällt oft aus, in: Böckler Impuls 20, S. 2

Jansen, R. (2000): Arbeitsbedingungen, Arbeitsbelastungen und Veränderungen auf betrieblicher Ebene, in: Dostal, W./Jansen, R./Parmentier, K. (Hrsg.): Wandel der Erwerbsarbeit: Arbeitssituation, Informatisierung, berufliche Mobilität und Weiterbildung, Nürnberg, S. 39-65 Janßen, D./Nachreiner, F. (2004): Flexible Arbeitszeiten, Bremerhaven Karasek R./Theorell, T. (1990): Healthy Work: Stress, Productivity, and the Reconstruction of Working Life, New York

Knesebeck, O. v.d./Klein, J./Grosse Frie, K./Blum, K./Siegrist, J.

(2010): Psychosoziale Arbeitsbelastungen bei chirurgisch tätigen Krankenhausärzten. Ergebnisse einer bundesweiten Befragung, in: Deutsches Ärzteblatt 14, S. 248-253

Lehndorff, S./Jansen, A./Kümmerling, A. (2009): Arbeitszeiten wieder so lang wie vor 20 Jahren. IAQ/HBS Arbeitszeit-Monitor seit 2001:

Auf Personalabbau folgten Arbeitszeitverlängerungen, IAQ-Report 1,

Duisburg/Essen

Lenhardt, U./Kuhn, J./Reusch, J. (2010): Die Arbeitswelt von heute: Daten, Schwerpunkte, Trends, in: Schröder, L./Urban, H.-J. (Hrsg.): Gute Arbeit. Handlungsfelder für Betriebe, Politik und Gewerkschaften, Frankfurt a.M., S. 429-491

Mergner, U. (1989): Zur sozialen Konstitution psychischer Belastung durch Arbeit, in: Zeitschrift für Arbeits- und Organisationspsychologie 2, S. 64-72

Natali, E./Deitinger, P./Rondinone, B./lavicoli, S. (2008): Exploring Stakeholders' Perceptions on Social Policies, Infrastructures and Social Dialogue in Relation to Psychosocial Risks, in: Leka, S./Cox, T. (Hrsg.): The European Framework for Psychosocial Risk Management: PRIMAEF, Nottingham, S. 79-95

Paoli, P. (1992): First European Survey on the Work Environment 1991-1992, Luxembourg

Paoli, P. (1997): Second European Survey on Working Conditions, Dublin

Pejtersen, J.H./Kristensen, T.S. (2009): The development of the psychosocial work environment in Denmark from 1997 to 2005, in: Scandinavian Journal of Work, Environment \& Health 4, S. 284-293 Pröll, U./Gude, D. (2003): Gesundheitliche Auswirkungen flexibler Arbeitsformen, Bremerhaven

Siegrist, J. (1996): Soziale Krisen und Gesundheit, Göttingen Theorell, T. (1997): How will future worklife influence health?, in: Scandinavian Journal of Work, Environment \& Health 4, S. 16-22

Wirtz, A./Nachreiner, F./Beermann, B./Brenscheidt, F./Siefer, A. (2009): Lange Arbeitszeiten und Gesundheit, http://www.baua.de/ Publikationen/Fachbeitraege/artikel20.,xv=vt.pdf (Zugriff: 15.01.2010) 